\title{
EchoGéo
}

54 | 2020

Varia

\section{Le front pionnier agro-désertique égyptien, par- delà rural et ruralité ?}

\author{
Delphine Acloque
}

\section{OpenEdition}

12 Journals

Édition électronique

URL : https://journals.openedition.org/echogeo/20441

DOI : 10.4000/echogeo.20441

ISSN : 1963-1197

\section{Éditeur}

Pôle de recherche pour l'organisation et la diffusion de l'information géographique (CNRS UMR 8586)

Référence électronique

Delphine Acloque, "Le front pionnier agro-désertique égyptien, par-delà rural et ruralité ? », EchoGéo [En ligne], 54 | 2020, mis en ligne le 31 décembre 2020, consulté le 26 août 2021. URL : http:// journals.openedition.org/echogeo/20441; DOI : https://doi.org/10.4000/echogeo.20441

Ce document a été généré automatiquement le 26 août 2021.

EchoGéo est mis à disposition selon les termes de la licence Creative Commons Attribution - Pas d'Utilisation Commerciale - Pas de Modification 4.0 International (CC BY-NC-ND) 


\title{
Le front pionnier agro-désertique égyptien, par-delà rural et ruralité?
}

\author{
Delphine Acloque
}

\section{Introduction}

Dès l'arrivée au pouvoir des Officiers libres en 1952 et parallèlement à la réforme agraire mise en œuvre dans les terres de la vallée et du delta du Nil, les périphéries désertiques deviennent le lieu de projection de l'avenir de l'agriculture du pays. En témoigne le lancement, dès 1954, du projet de la province de Tahrir (libération) au sein des marges occidentales du Delta. Une véritable frontière agro-désertique émerge alors et se déplace de plus en plus loin vers l'ouest et le sud du désert occidental dans des environnements aux contraintes croissantes, tant du point de vue des distances et de la logistique, de la main-d'œuvre que de l'accès à l'eau. Ces terres arides s'imposent ainsi progressivement comme le laboratoire d'une agriculture en rupture - réelle et/ou revendiquée - avec les anciennes terres (Ireton, 2011) et avec la paysannerie traditionnelle, ceci au profit d'une classe d'agriculteurs, d'entrepreneurs privés, de techniciens ou encore d'experts agricoles (Aboulnaga et al., 2017).

Alors que les recherches contemporaines mettent en avant les nouvelles ruralités et s'attachent à penser le rural sans l'agricole au Nord comme au Sud (Rieutort, 2012 ; Pesche D. et al., 2016), le cas du front pionnier agro-désertique égyptien nous invite à penser l'agricole sans le rural. Dix ans après la publication de l'ouvrage de H. Ayeb (2010) qui s'interrogeait sur la fin du fellah (paysan) égyptien, cet article se propose d'éclairer la nature des nouveaux territoires agro-désertiques égyptiens et de les analyser sous l'angle de leur rapport au rural et à la ruralité. Celle-ci peut être définie comme l'ensemble des «modes d'habiter" (Mathieu, 2014), où le lien à l'environnement, aux patrimoines, aux paysages agraires et forestiers revêt une importance particulière.

Dénonçant la confusion entre rural et agricole, la géographie contemporaine, et plus largement les sciences sociales, ont notamment cherché à affranchir la ruralité de son 
carcan agricole, en analysant la diversification de ses activités et de ses acteurs. Ceux-ci se caractérisent par des pratiques, des sociabilités et des formes d'attachement renouvelées aux territoires (Lévy et Lussault, 2003 ; Margetic et Rouget, 2019). Or, dans les secteurs de bonification - comme dans la plupart des fronts pionniers à l'échelle de la planète (Arnauld de Sartre, 2006)-, ce sont justement ces pratiques et cet attachement singulier à la terre et aux espaces mis en valeur qui font problème et constituent l'enjeu problématique de cet article. Faut-il s'affranchir du concept de ruralité pour rendre compte des espaces du front pionnier? Quelles catégories sociospatiales utiliser ou construire afin de caractériser ces néo-territoires agrodésertiques ? Il s'agit par là-même de prendre part à la réflexion plus large sur les liens entre agriculture et territoire (Albaladejo, 2012 ; Guibert et al. 2015 ; Morgan et al., 2008 ; Rieutort, 2009). Les enjeux du rapport au territoire de la nouvelle agriculture égyptienne et de ses acteurs se déclinent à différentes échelles: des parcelles de petits paysans, regroupées au sein de villages créés ex-nihilo, aux vastes exploitations agrocapitalistes contemporaines, parfois distantes de plusieurs centaines de kilomètres des principaux espaces de peuplement.

L'article s'appuie sur des enquêtes de terrain réalisées de 2012 à 2016 dans les secteurs de nouvelles terres représentatifs des phases successives de bonification, notamment au sein des marges arides du Delta du Nil (illustration 1). Les marges occidentales du Delta se caractérisent plus particulièrement par un déplacement continu de la frontière de plus en plus loin au sein du désert libyque, ceci des années 1950 à aujourd'hui. Afin de comprendre le système d'acteurs et d'intérêts impliqués dans la construction de nouveaux territoires agro-désertiques, environ 80 entretiens ont été menés auprès des différents acteurs du processus de bonification : petits et moyens producteurs; grandes entreprises de l'agrobusiness et exportateurs; propriétaires fonciers citadins souvent pluriactifs ; experts nationaux et internationaux. 
Illustration 1 - Terrains d'enquête au sein des marges désertiques du Delta du Nil

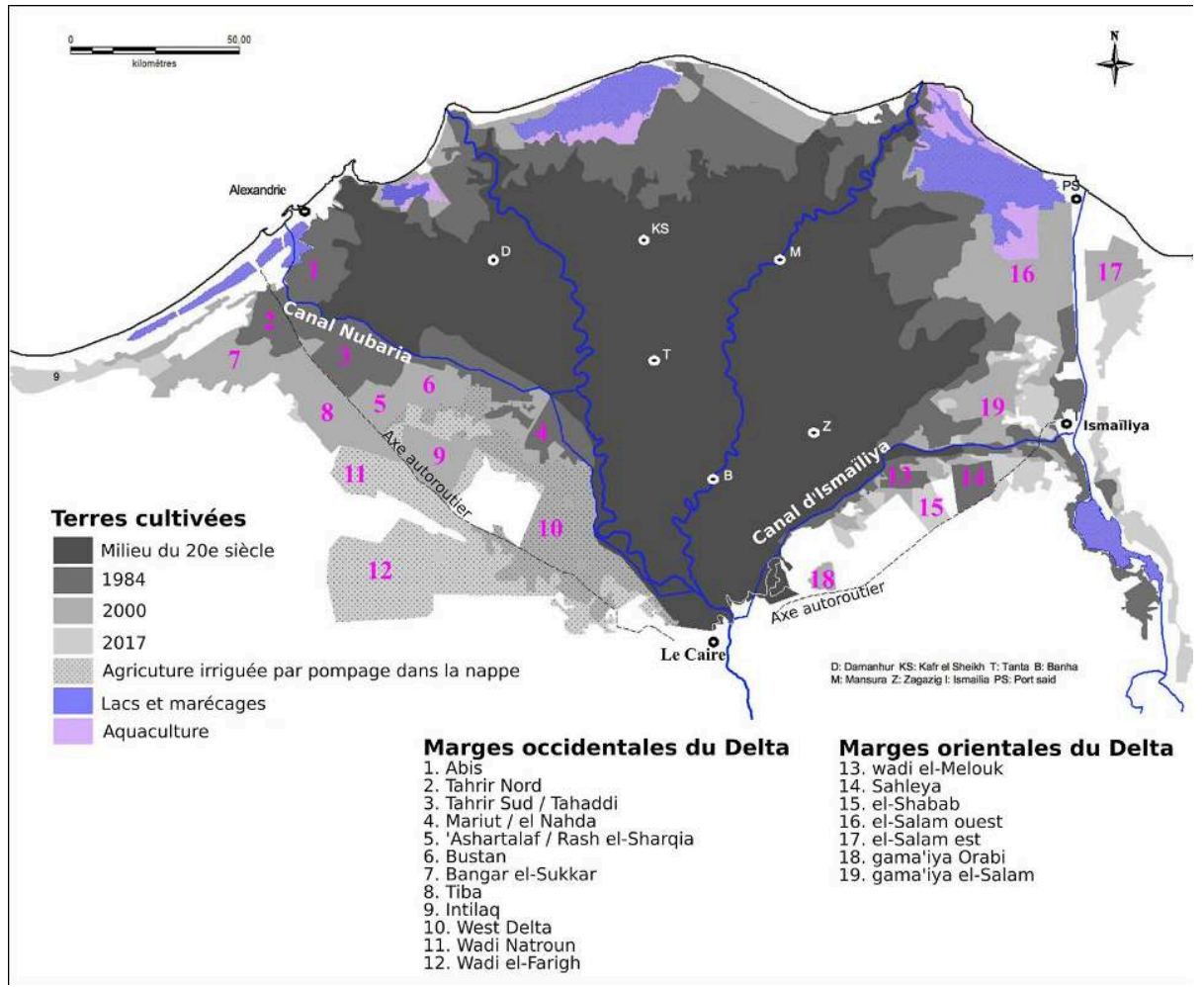

Auteurs : Molle, 2019 ; Acloque, 2019

Après une première section dédiée à la contextualisation du front pionnier, à ses acteurs et aux discours qui l'ont façonné, l'article se propose d'analyser la frontière agro-désertique et le processus de construction territoriale à la lumière de la notion de ruralité et de sa "mise à l'épreuve». Une typologie combinant critères environnementaux, sociaux, productifs et politico-économiques permet de distinguer quatre types d'espaces agro-désertiques, qui s'organisent selon un gradient décroissant de ruralité.

\section{Construire l'avenir de l'agriculture égyptienne dans les marges désertiques}

\section{Justifier et revendiquer la rupture avec l'ancien monde}

Tableau 1 - Les registres de justification de la conquête agro-désertique par les acteurs politiques et institutionnels

\begin{tabular}{|l|l|}
\hline Registre de justification & Discours et arguments mobilisés \\
\hline Impératif démographique & $\begin{array}{l}\text { Créer de nouvelles communautés de peuplement pour } \\
\text { échapper à l'hyper-densité des anciennes terres et au } \\
\text { morcellement croissant des parcelles agricoles }\end{array}$ \\
\hline
\end{tabular}




\begin{tabular}{|l|l|}
\hline Justification sanitaire & $\begin{array}{l}\text { Promouvoir la virginité des terres désertiques, l'air pur et } \\
\text { l'eau saine qui permettent la satisfaction des exigences } \\
\text { internationales en termes de qualité des produits }\end{array}$ \\
\hline $\begin{array}{l}\text { Idéal de la modernité et de la } \\
\text { modernisation }\end{array}$ & $\begin{array}{l}\text { Valoriser les infrastructures (stations de pompage/canaux) } \\
\text { et les innovations techniques } \\
\text { Promouvoir une société d'agriculteurs modernes }\end{array}$ \\
\hline $\begin{array}{l}\text { Développement économique et } \\
\text { soupape sociale }\end{array}$ & $\begin{array}{l}\text { Encourager la croissance du secteur agro-industriel et des } \\
\text { exportations } \\
\text { Créer des emplois, notamment pour les jeunes diplômés }\end{array}$ \\
\hline Sécurité alimentaire de la population & $\begin{array}{l}\text { Produire davantage via la mise en culture de nouvelles } \\
\text { terres } \\
\text { Exporter des produits à haute valeur ajoutée et importer } \\
\text { l'alimentation de base de la population (blé notamment) }\end{array}$ \\
\hline $\begin{array}{l}\text { Développement durable (années } \\
\text { hydrique (années 2010) }\end{array}$ & $\begin{array}{l}\text { Maximiser la valorisation des ressources en eau dans un } \\
\text { contexte de rareté } \\
\text { Promouvoir l'efficacité de l'irrigation moderne (pivot ; } \\
\text { goutte-à-goutte) }\end{array}$ \\
\hline
\end{tabular}

Sources : Mitchell, 2002 ; Scott, 1998 ; Sims, 2014 ; Sowers, 2011.

Résumés dans le tableau ci-dessus, différents registres de justification ont été mobilisés, combinés et reformulés par les institutions égyptiennes au cours des six décennies de conquête agricole du désert jusqu'à aujourd'hui. Tous s'inscrivent dans une volonté affichée de moderniser le pays et ses campagnes : le processus de bonification doit y contribuer en faisant du désert le lieu de construction d'une nouvelle Egypte productive tournant le dos à la vallée, à son territoire et à sa société "chaotiques", ainsi qu'à sa paysannerie décrite comme immuable, voire comme un obstacle à la modernisation (Ayrout, 1938 ; Critchfield, 1978). Anouar el-Sadate (Président égyptien, 1970-1981) s'interrogeait ainsi : «Pourquoi ne pas nous extirper de cette étroite vallée pour atteindre de nouveaux horizons au sein de vastes terres riches en eau?» (The World, 1978). On retrouve ici la volonté de «rupture radicale avec l'histoire et la tradition" (Scott, 1998, p. 93) caractéristique du high modernism. Cette idéologie, qui trouve son origine dans les pays occidentaux comme sous-produit du positivisme et du progrès, se diffuse au cours de la seconde moitié du XXe siècle au sein de nombreux pays du Sud. Dans le cas égyptien, elle s'accompagne d'une volonté de rupture spatiale : le nouveau projet territorial ne vise pas à « réécrire » l'agriculture et le développement du pays sur les terres du passé, mais à « relocaliser physiquement la paysannerie dans un nouvel environnement naturel, social et culturel » (Scott, 1998). Les gouvernements égyptiens successifs cherchent non seulement à dépasser la contrainte spatiale que représente l'étroitesse de la Vallée et du Delta du Nil, mais plus largement à surmonter les «plaies » qui menaceraient à la fois le bien-être de la population et l'économie du pays. Par contraste, les espaces désertiques ont fait l'objet de discours de valorisation célébrant la virginité de la terre, de l'air et de l'eau. 


\section{Terres nouvelles et société nouvelle " dé-paysanisée » (Van der Ploeg, 2018)}

Dès les années 1950, une société nouvelle est promue sur ces terres. Celle-ci doit être constituée d'une forme d'avant-garde capable d'utiliser les innovations techniques les plus performantes et de se libérer de tous les carcans précédents. En témoignent la toponymie et le lexique utilisés, insistant sur la rupture et l'affranchissement avec les terres anciennes, à l'image du projet de Tahrir (« libération » en arabe), pour lequel les colons ont dû accepter six mois de formation préalable pendant lesquels ils ont été complètement pris en charge par l'État pour le logement, l'alimentation et l'habillement, tandis que les visites de la famille étaient interdites (Fanchette, 1997). M. Hassanein, en charge de ce projet, proclamait alors : «Tahrir ne fait pas que planter, cultiver et récolter, mais a révolutionné les relations sociales. Tahrir a dépassé, avec les paysans, 5000 ans d'exploitation et de dégradation pour une nouvelle vie » (Fanchette, 1997, p. 125). Ainsi, conformément aux logiques de front pionnier analysées ailleurs dans le monde (Monbeig, 1952 ; Théry, 1976 ; Arnauld De Sartre, 2006 ; Marshall, 2009), les objectifs initiaux, qui impriment encore largement les projets contemporains, ont été de construire un espace neuf pour des agro-pionniers à la pointe de la modernité. Si le sens et la nature de cette modernité ont évolué au gré des mutations technologiques, agronomiques, politico-économiques ou idéologiques de l'Égypte depuis les années 1950 (Mitchell, 2002), elle s'accompagne d'une forme de "standardisation » croissante des producteurs (Scott, 1998, p. 345). Ceux-ci doivent être modelés pour créer des agriculteurs égyptiens modernes et participer à l'avènement d'une agriculture entrepreneuriale. C'est ainsi un processus de dé-paysanisation qui est promu. Celui-ci a notamment été décrit par Van der Ploeg (2018) comme un projet intellectuel et idéologique d' "adieu définitif aux pratiques comme aux identités paysannes ». Initié dans l'Europe des années 1960 et fondé sur l'usage de dichotomies simplificatrices (tradition-modernité ; petit-grand ; paysan-entrepreneur ; vivrier-commercial), ce projet s'est diffusé vers les pays du Sud et a largement façonné les projets de développement rural de leurs gouvernements et des principaux bailleurs de fonds internationaux depuis les années 1990 (Van der Ploeg , 2018).

\section{Un front pionnier qui s'adapte aux impératifs politico-économiques}

Le projet politique de construction d'une société agricole d'avant-garde est initié dans le contexte de la chute de la monarchie et de la proclamation de la République égyptienne (1952). Alors que la période nassérienne (1952-70) est caractéristique du développementalisme et de la diffusion de révolutions socialistes dans le Tiers-Monde, la conquête de nouvelles terres agricoles revêt des modalités et des objectifs largement transformés avec l'ouverture économique de la fin des années 1970. Elle devient progressivement indissociable de la promotion de cultures à haute valeur ajoutée, destinées notamment à l'exportation, et de l'encouragement des initiatives privées, renforcée par les mesures d'ajustement structurel des années 1990.

Dans les années 1990-2000, en lien avec l'accélération du processus de libéralisation économique, une double-stratégie est alors mise en œuvre au sein des nouvelles terres : d'une part, la promotion d'un modèle agro-exportateur dominé par de grandes et très grandes exploitations et, d'autre part, la bonification de nouveaux secteurs dédiés en 
priorité à deux catégories de petits producteurs : les paysans dits « compensés » pour la perte de leurs terres suite à la loi de libéralisation du foncier, promulguée en 1992 et devenue effective en $1997^{1}$; et les jeunes diplômés à la recherche d'un emploi. Face à une situation économique dégradée, marquée par l'augmentation du chômage des jeunes et par les retours contraints de nombreux migrants égyptiens lors de la guerre du Golfe à l'été 1990, le régime du président Moubarak a en effet misé sur les terres désertiques pour fournir un emploi et un statut social à une partie de la jeunesse égyptienne. Le programme "des diplômés" est ainsi lancé en 1987. Initialement destiné aux diplômés des écoles d'agriculture, il est progressivement étendu à l'ensemble des diplômés de l'université, puis du secondaire, faisant de l'allocation de terres (et de l'activité agricole) une soupape de sécurité face à la pression sociale croissante. Vingt-cinq ans plus tard et dans le contexte de l'après-révolution de 2011, le président Sissi lance le projet de bonification $\mathrm{du}$ "million et demi de feddans ${ }^{2}$ »(illustration 2), censé créer deux millions d'emplois dans l'agriculture et l'agro-alimentaire (EEDC, 2015, p. 21). L'objectif pour le pouvoir est notamment de promouvoir une "élite» de producteurs, capable de s'adapter aux exigences des entreprises agro-industrielles ou agro-exportatrices et regroupés au sein d'un même secteur de bonification.

\section{Illustration 2 - Les secteurs de bonification du projet du « million et demi de feddans »}

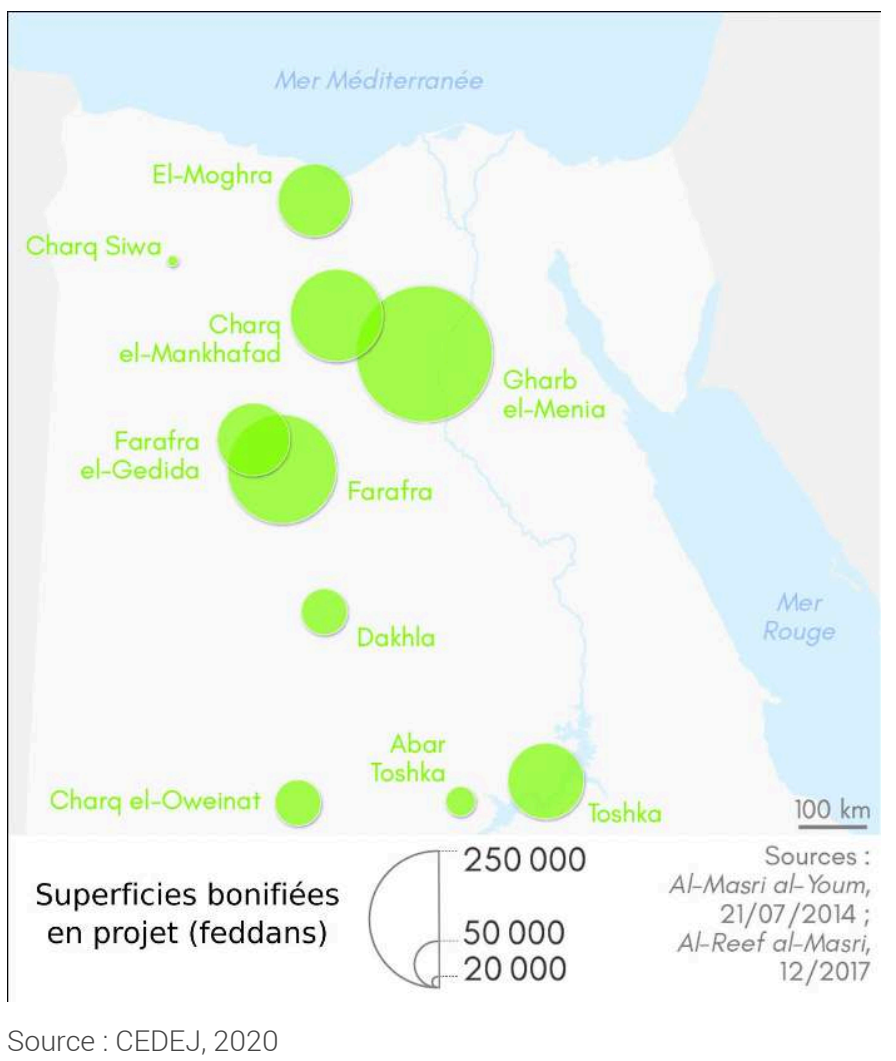

À côté des programmes gouvernementaux successifs, des dynamiques spontanées et non planifiées d'installation de producteurs de tailles variées - de l'exploitation de deux hectares à la firme agro-exportatrice à la tête de milliers d'hectares - s'accélèrent au sein des marges désertiques, ceci en dépit de la complexité des processus d'accès au foncier ${ }^{3}$. C'est notamment la diffusion de nouvelles techniques d'irrigation et l'usage 
des eaux souterraines qui ont permis l'extension du front pionnier au cours des deux dernières décennies, avec cependant des succès très inégaux qui dépendent de la capacité d'accès aux technologies et aux marchés les plus sélectifs.

\section{Le difficile bilan statistique de la conquête agro-désertique}

Illustration 3 - Évolution des superficies cultivées dans les nouvelles et anciennes terres

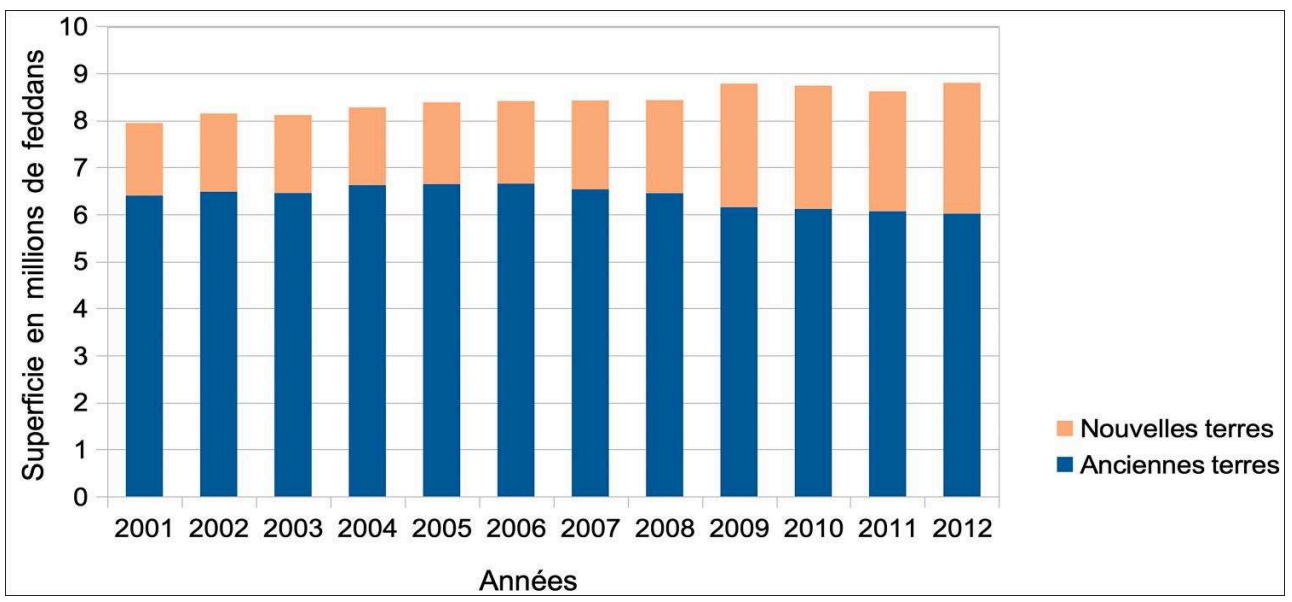

Source : CAPMAS, Le Caire, 2013.

D'après les données du CAPMAS ${ }^{4}$ pour la période 2001-2012, la superficie de nouvelles terres serait passée de 1,5 million de feddans en 2001 à environ 2,8 millions en 2012 (illustration 3). Ces chiffres, débattus au sein même des instances gouvernementales, doivent être considérés avec beaucoup de précaution. La difficulté à obtenir des statistiques précises et fiables tient notamment à l'expression même de terres bonifiées (al-aradi al-mustaslaha), qui recouvre une grande variété de situations. Si une partie de ces terres est effectivement cultivée et produit des revenus parfois très conséquents, une autre partie est en attente de mise en culture; d'autres encore sont plantées mais produisent à perte ou marginalement; enfin, certaines parcelles désertiques auparavant cultivées sont laissées à l'abandon. Les raisons en sont multiples : coût trop élevé de la mise en culture, échec des plantations, processus de mise en vente, attente spéculative, salinisation des terres et infertilité. En outre, peu de bilans chiffrés s'appuient sur des relevés de terrain détaillés. Au total, au tournant des années 2010, la part de nouvelles terres est évaluée dans une fourchette très large entre $15 \%$ et $30 \%$ de la superficie cultivée totale (Aboul-Naga et al., 2017 ; Najjar, 2013)

Si les nouvelles terres regroupent moins de $5 \%$ des exploitations agricoles (Aboulnaga et al., 2017 ; Kassim et al., 2018), elles expliquent toutefois en grande partie la forte hausse des exportations agricoles égyptiennes des dix dernières années ${ }^{5}$ et les grandes exploitations des terres désertiques représenteraient environ 15\% de la production agricole totale du pays (EEDC, 2015). Ce chiffre est bien supérieur dans le cas de productions fruitières et maraîchères (notamment les raisins, pommes, fraises, agrumes ou pommes de terre). Loin de viser à l'exactitude statistique dans l'évaluation de la contribution à l'économie nationale ou à la superficie cultivée de la nouvelle agriculture, cet article se propose d'éclairer les recompositions socio-spatiales et les modalités de construction territoriale associées à la mise en culture des marges arides. Différents secteurs de terres désertiques sont concernés, donnant naissance à une 
pluralité de nouveaux territoires agricoles que la section suivante se propose de caractériser et d'analyser.

\section{Nouvelles terres et fin de la ruralité ?}

\section{Front pionnier et nouveau secteur agro-capitaliste}

Afin de mieux comprendre la nature des espaces agro-désertiques, il convient d'approfondir la définition de la ruralité à la lumière des transformations agraires des dernières décennies. Les enjeux liés à l'émergence de nouveaux acteurs agricoles et la question de leur rapport à la terre - en Égypte comme dans de nombreux pays du Sud s'inscrivent dans une réflexion plus large sur les transformations des espaces ruraux, le devenir de la petite paysannerie dans un contexte néo-libéral (Akram-Lodhi et Kay, 2009) et les enjeux de la précarisation voire de la prolétarisation de celle-ci, décrits et analysés par les chercheurs des agrarian studies (Bernstein, 2001 ; Araghi, 2009). Les mutations contemporaines de l'agriculture égyptienne, à l'œuvre au sein des espaces de bonification désertique, participent de l'expansion du secteur de l'agrobusiness et de la montée en puissance d'un capitalisme agricole extractiviste (Svampa, 2019). Ces modèles reposent notamment sur le rôle croissant des investisseurs extra-ruraux - qui interviennent à divers niveaux des filières productives -, ainsi que sur le recours constant aux innovations agronomiques et technologiques pour l'irrigation et la production, elles-mêmes à l'origine de rapports renouvelés aux territoires.

\section{Le processus de construction territoriale à la frontière}

Les espaces du front pionnier égyptien ont été conçus comme des espaces dont la vocation première est la production agricole et agro-alimentaire. Il apparait en effet clairement, dans la pratique des politiques de bonification, que les gouvernements successifs jusqu'à aujourd'hui ont largement réduit la ruralité à la notion de productivité, envisageant la construction de communautés productives plutôt que de sociétés rurales au sens plein. En témoigne la primauté qui a été donnée aux infrastructures d'irrigation, au bâti ou encore au découpage parcellaire par comparaison aux services à la population et aux dimensions sociales de la vie quotidienne. Autre élément très significatif de la priorité accordée à la fonction de production agricole : c'est la coopérative agricole des villages des nouvelles terres qui a souvent constitué le premier et le principal lieu de sociabilité, ainsi que le relais de la présence publique dans les marges désertiques. En outre, de nombreux secteurs bonifiés se caractérisent par une absence d'autonomie administrative ou par un transfert très limité de pouvoirs, engendrant une marginalisation renforcée de ces territoires et de leurs habitants. Pour ces derniers, la construction d'une identité et d'une appartenance territoriale est ainsi d'autant plus longue et difficile. La croissance rapide de la population de certains secteurs de nouvelles terres ${ }^{6} \mathrm{n}^{1} \mathrm{a}$ en effet pas été suivie par le développement concomitant des structures administratives. À l'échelle nationale, plus de 4000 communautés étaient situées en dehors du système administratif et représentatif local au tournant des années 2010 (Martinez-Vazquez et Timofeev, 2011, p. 394). Dans le cas de Tahrir Sud, pourtant parmi les secteurs bonifiés les plus anciens, un markaz (équivalent du district ou de la commune) n'a été créé qu'au 
cours des années 2000 afin d'assurer l'encadrement administratif de cette région et sa représentation au Parlement.

Toutefois, les secteurs les plus anciens se distinguent par un processus de maturation sociale et économique, les plaçant sur une trajectoire de normalisation et d'intégration croissante aux espaces voisins. La dimension temporelle est ainsi nécessaire pour comprendre la différenciation interne des territoires du front pionnier. Elle n'est cependant pas suffisante et il est nécessaire de combiner un ensemble de données historiques, politiques, environnementales et socio-économiques. La frontière se caractérise en effet par une pluralité d'espaces agro-productifs et par des rapports différenciés à l'espace selon les secteurs de nouvelles terres. C'est l'objet de la section suivante qui propose la construction d'une typologie.

\section{Nouveaux territoires agricoles et gradient de ruralité}

Afin d'éclairer la nature et les caractéristiques des espaces agro-désertiques, nous proposons de construire une typologie à partir des données recueillies au cours des enquêtes auprès de différentes catégories de producteurs et d'exploitations: allocataires de programmes gouvernementaux; petits locataires; entrepreneurs; firmes. Ces données permettent de définir un ensemble de critères répartis selon deux axes principaux sur l'organigramme ci-dessous (illustration 4). L'axe horizontal regroupe les quatre critères suivants: l'ancienneté du processus de bonification contribuant au degré de maturité du secteur concerné ; la distance vis-à-vis de la vallée et du delta du Nil, à laquelle sont associées des contraintes inégales en termes de logistiques et d'installation des activités et populations; les densités de population et l'existence d'une trame villageoise en lien avec des projets gouvernementaux de peuplement; et enfin, la diversité des activités et fonctions. L'axe vertical, dédié à la nature des activités agricoles et aux modalités de production, s'organise quant à lui en deux pôles opposés en fonction de trois critères: nature et destination des productions ; modalités d'irrigation ; main-d'œuvre. 
Illustration 4 - Typologie des espaces agro-désertiques égyptiens et gradient de ruralité

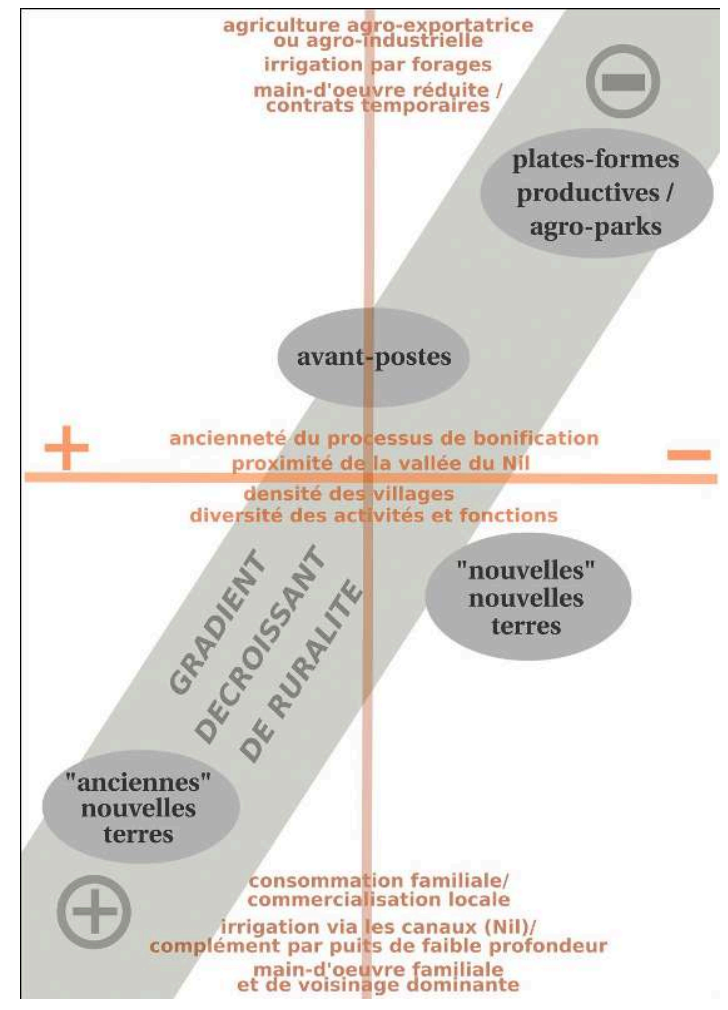

Le positionnement différencié des espaces enquêtés sur ces deux axes permet alors l'identification de quatre types principaux de territoires agro-désertiques, répartis selon un gradient décroissant de ruralité :

1. Les « anciennes " nouvelles terres en voie d'assimilation : bonifiés entre les années 1950 et 1970, ces secteurs situés au contact direct avec les terres du Delta se distinguent par une intégration croissante aux espaces historiquement mis en valeur. Ils incarnent ce que l'on peut désigner comme la greffe réussie d'une ruralité transférée ;

2. Les «nouvelles» nouvelles terres des marges du Delta: bonifiées depuis les années 1990, elles associent secteurs dédiés aux petits producteurs établis dans le cadre de projets gouvernementaux et grandes entreprises agricoles. Elles se caractérisent par un processus de maturation et de territorialisation en cours ;

3. Les avant-postes: établis de façon spontanée par des pionniers - individus ou sociétés -, ils sont situés en dehors des secteurs de planification, tout en étant majoritairement dans la continuité des espaces déjà bonifiés ;

4. Les plates-formes productives ou agro-parks: symboles d'une modernisation agricole fondée sur l'innovation productive et technologique, ces espaces sont au cœur des projets contemporains et constituent un objectif à atteindre pour les nouveaux espaces bonifiés identifiés sur la carte nationale du « million et demi de feddans ».

Les « anciennes » nouvelles terres : une ruralité en transfert ? 
Illustration 5 - Le Canal Nubaria entre anciennes terres du Delta et « anciennes » nouvelles terres

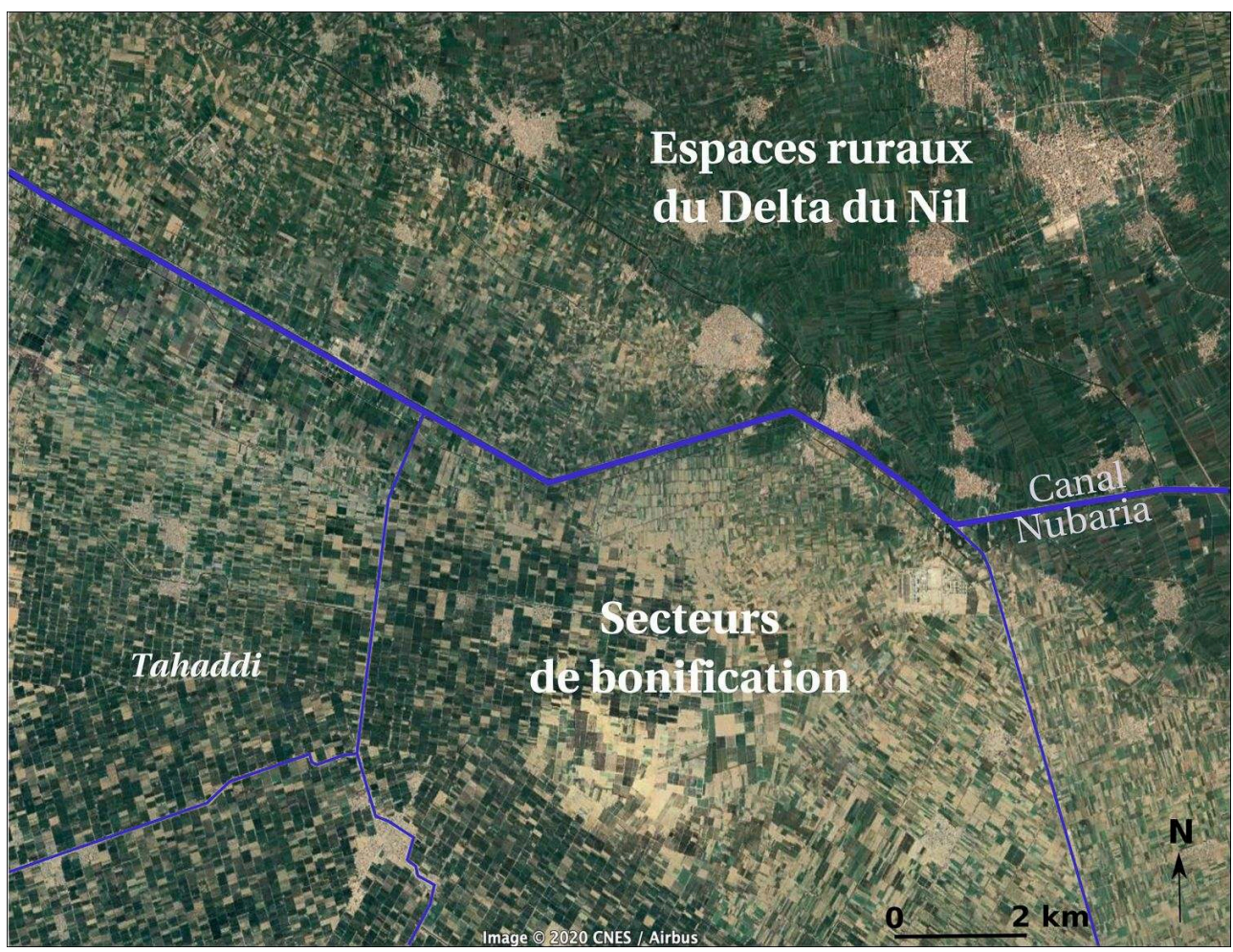

Source : Image Google Earth, 2020.

Cette image satellitaire de l'ouest du delta du Nil (illustration 5) met en avant une distinction entre, d'une part, les paysages et les densités agricoles et villageoises des terres historiques situées au Nord-Est et, d'autre part, le reste du territoire et plus particulièrement le sud du canal Nubaria. Constitué de secteurs qui ont été bonifiés depuis les années 1950-60, cet espace est désigné par l'expression "anciennes » nouvelles terres. En témoigne le cas du secteur de Tahaddi (Sud-Ouest de l'image) qui présente une organisation agraire singulière par la géométrie des parcelles, leur substrat sableux et les paysages productifs. Ce secteur de terres bonifiées est cependant très proche des anciennes terres voisines, auxquelles il est connecté sous de nombreux aspects. Espaces de bonification anciens et espaces contigus du Delta se caractérisent en effet par une diversité de relations sociales et économiques, qui prennent la forme d'échanges de produits, de capitaux, de savoirs agronomiques, ou encore de pratiques et de mobilités quotidiennes. Des formes de continuité territoriale, ainsi que des identités et sociabilités communes, permettent ainsi de conclure à une même ruralité partagée entre anciennes terres et « anciennes » nouvelles terres.

Plusieurs entretiens ont par ailleurs montré que la maturation du front pionnier s'accompagne d'un processus d'autonomisation et de normalisation des secteurs bonifiés. C'est ainsi le cas d'Ibrahim, qui réside dans le secteur de 'Ashartalaf (bonifié à partir des années 1970) et mentionne que "[s]a vie est là » et qu'« il y a tout ici » (entretien, 2014). Aujourd'hui propriétaire de 10 feddans et locataire d'une superficie de 50 feddans auprès d'un propriétaire foncier citadin, il produit des cultures traditionnelles et des pommes de terre sous pivot destinées à l'agro-industrie locale. La relocalisation d'une partie des grossistes au cœur des secteurs de bonification des 
marges du Delta semble également consacrer l'intégration croissante de ceux-ci aux dynamiques rurales.

\section{Les « nouvelles » nouvelles terres : une ruralité en construction}

Les «nouvelles » nouvelles terres désignent ici les secteurs bonifiés à partir des années 1990-2000, prioritairement dans les marges occidentales du Delta. Le Mubarak national resettlement scheme for the graduates (1987) a consisté à attribuer des terres désertiques, sous la forme de lots de 5 à 10 feddans destinés à une petite agriculture familiale. Environ $25 \%$ des terres nouvelles attribuées entre les années 1980 et le milieu des années 2000 l'ont été dans le cadre de ce programme (Banque mondiale, 2006). Les secteurs de Tiba et Intilaq, respectivement situés à l'Ouest et à l'Est de l'axe autoroutier Alexandrie-Le Caire (Illustration 1), sont emblématiques de ces nouvelles communautés agro-désertiques établies le long de canaux de dérivation des eaux du Nil (illustration 6). Avec environ 7800 attributaires à Tiba et 9700 à Intilaq, celles-ci sont organisées en villages créés ex-nihilo, où chaque allocataire a reçu une maison de deux pièces et une parcelle de terres sous la forme d'un prêt sur trente ans.

Illustration 6 - Le secteur de bonification de Tiba et son organisation territoriale

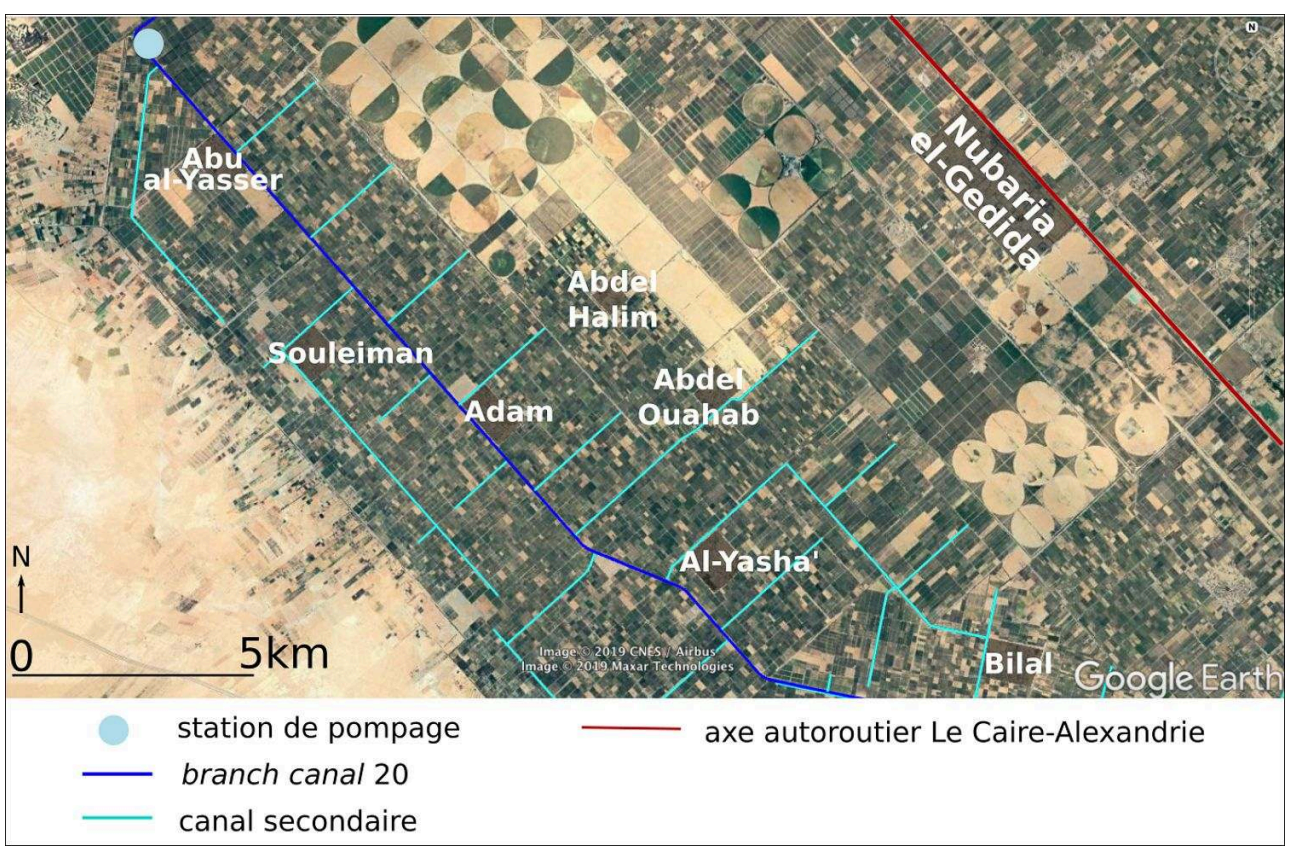

Source : Image GoogleEarth, 2019. Réalisation : Acloque, 2020.

Les modes de vie et les pratiques qui se construisent dans ces communautés nouvelles s'analysent à la lumière de contraintes spécifiques: eau (accès irrégulier à l'eau d'irrigation, eau impropre à la consommation selon certaines familles rencontrées); coût élevé de l'électricité, indispensable au fonctionnement des pompes pour l'irrigation; surcoût des produits de base et des intrants agricoles ; manque de services à la population. Toutefois, plusieurs petits producteurs du secteur de Tiba, qui ont réussi à pérenniser leur exploitation après des débuts difficiles et à faire venir leur famille depuis les anciennes terres du Delta, affirment que "tout est mieux ici", insistant en particulier sur «l'espace, la propreté, l'air plus sain» (entretien, Tiba, 2014). 
La solidarité importante entre les allocataires des programmes gouvernementaux, qui se sont tous installés à la même période et qui pour certains proviennent des mêmes villages d'origine, est également fréquemment évoquée pour rendre compte de nouveaux liens sociaux, ainsi que des échanges de pratiques et de savoirs au sein des secteurs bonifiés. C'est notamment le cas pour la catégorie des bénéficiaires. Il s'agit majoritairement de petits paysans du Delta qui ont été contraints de quitter leur terre en raison de la hausse des loyers fonciers et des difficultés croissantes d'accès à la terre. La plupart d'entre-eux mentionne le rôle décisif des solidarités de voisinage et d'un processus collectif d'imitation-apprentissage, à l'image de la conversion progressive de leurs nouvelles terres en vergers d'agrumes ou de raisins.

En outre, ces espaces de bonification ont été marqués par des mobilités nombreuses (départs et arrivées de nouveaux producteurs) et par un ensemble de transactions foncières (Alary et al., 2018). Celles-ci sont à l'origine d'une diversification croissante des profils socio-économiques des résidents, avec le rachat ou la location de terres par des agriculteurs extérieurs aux programmes gouvernementaux ou encore des processus d'agrandissement des exploitations. Par ailleurs, l'agriculture ne semble pas être le seul horizon professionnel des habitants des secteurs bonifiés. C'est notamment le cas de la deuxième génération, désormais parvenue à l'âge adulte. Un producteur installé à Tiba à la fin des années 1990 explique ainsi que l'un de ses fils est devenu chauffeur, alors que l'autre est commerçant de détail dans leur village (entretien, Bangar el-Sukkar, 2014). On assiste donc progressivement à la diversification du tissu socio-économique local au-delà de la seule production agricole et à la densification des réseaux de sociabilité, tous deux caractéristiques d'une ruralité en construction.

Toutefois, la réalité de l'organisation interne de ces nouveaux villages est sommaire avec une voirie non stabilisée (illustration 7) et des équipements qui demeurent très en-deçà des besoins des populations. En témoignent les architectures fantômes de bâtiments publics, le manque d'infrastructures pour les marchés hebdomadaires et la pénurie de transports. Ces espaces sont notamment confrontés aux contraintes logistiques importantes, au manque de lieux de sociabilité, ainsi qu'à la difficulté de trouver des professionnels de santé ou d'éducation acceptant de s'y installer. Les services à la population ont par ailleurs pâti, à partir des années 1990, du retrait plus global de l'État, ainsi que des modalités d'intervention des agences de développement rural, dont les projets sont par nature à durée limitée. 
Illustration 7 - Village du secteur de Tiba : inachèvement et faiblesse des équipements

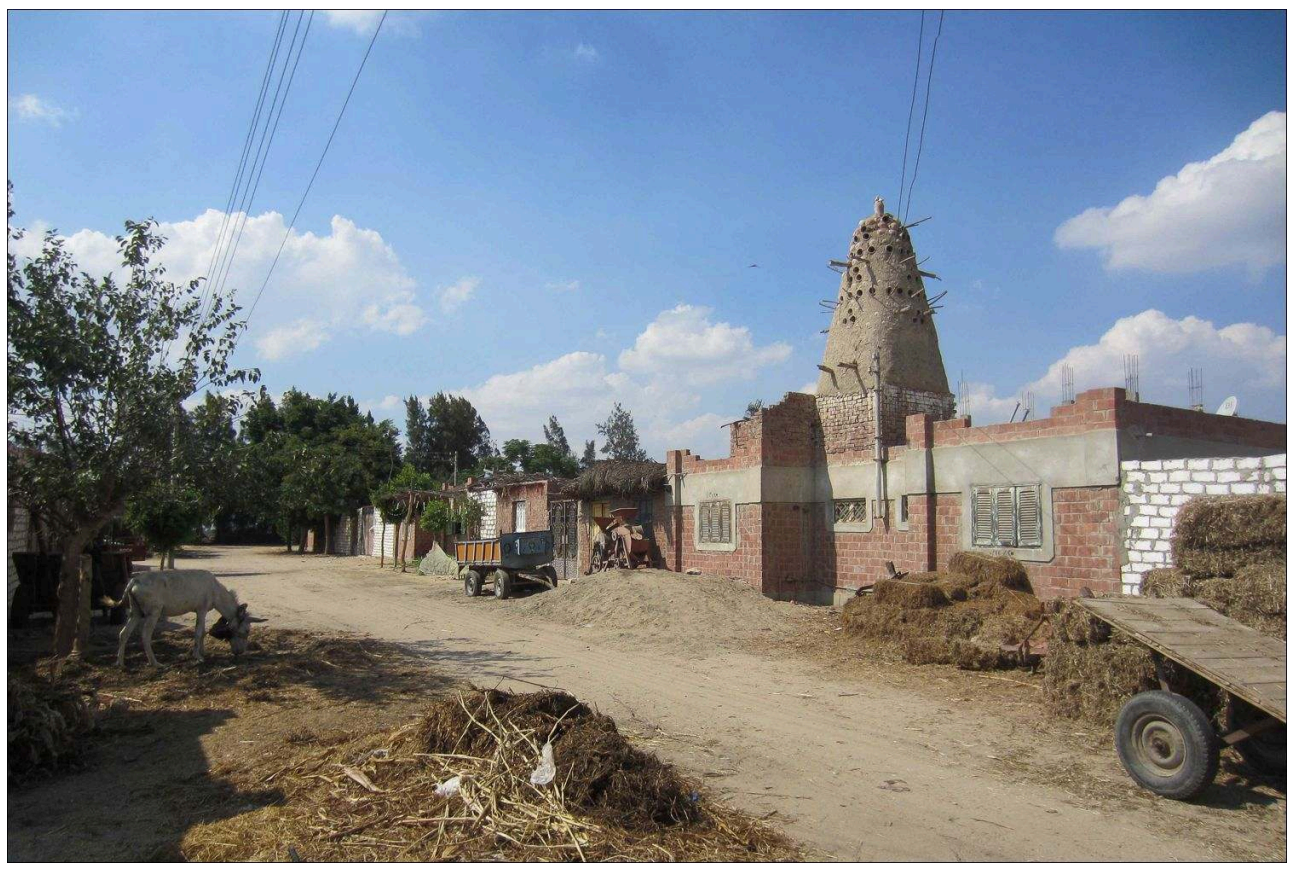

Auteur : D. Acloque, 2014

\section{Les avants-postes : lieux de projection du front pionnier}

Illustration 8 - Front pionnier et avant-postes du secteur de Wadi Natroun

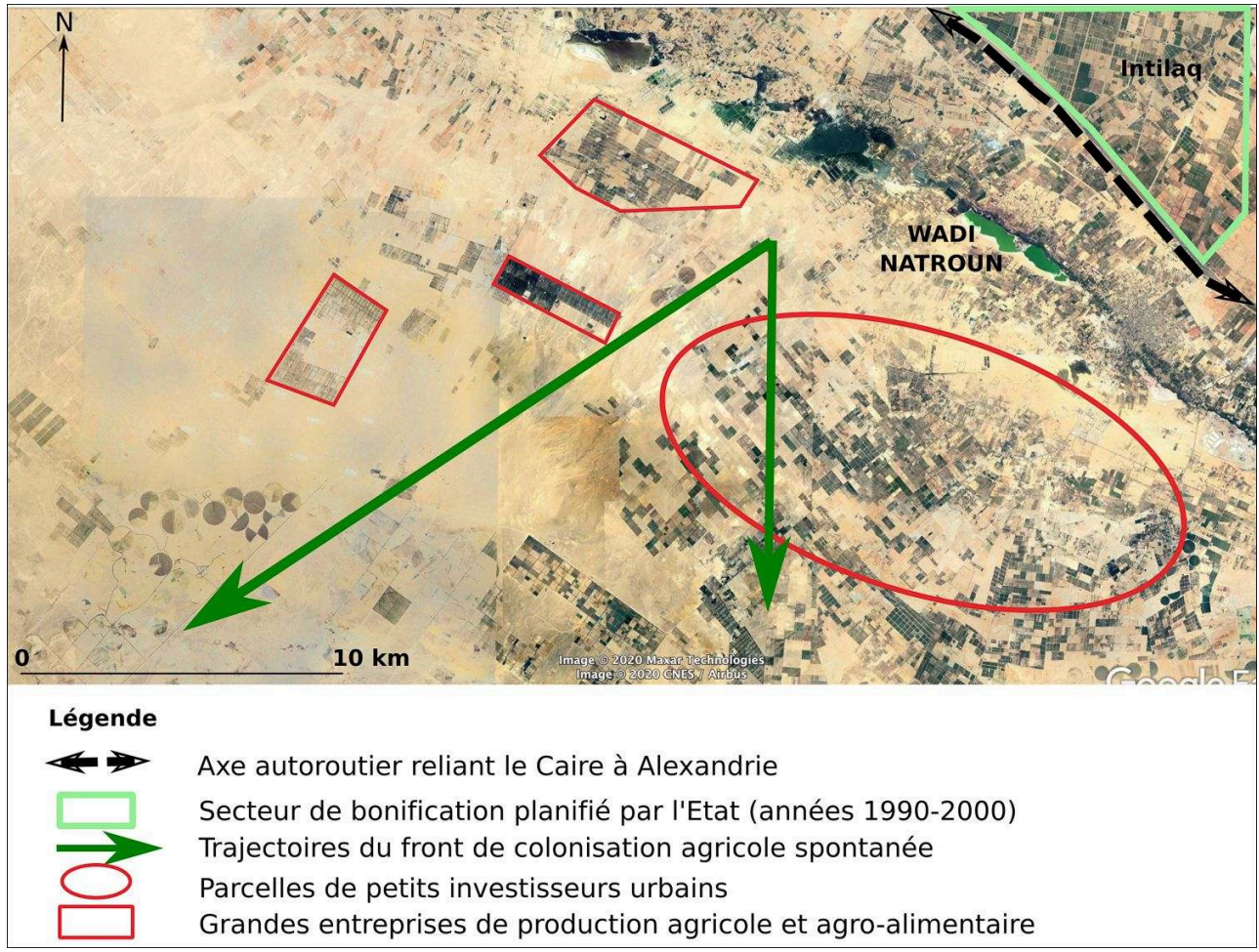

Source : Image GoogleEarth, 2020. Réalisation : D. Acloque, 2020. 
S'inscrivant dans le champ lexical des fronts - qu'ils soient pionniers ou militaires -, la notion d'avant-poste désigne ici un ensemble d'espaces agricoles en position avancée, et éventuellement détachée, par rapport à la continuité des terres historiquement mises en valeur ou des secteurs agro-désertiques déjà existants. Ces avant-postes sont délimités spatialement par les parcelles mises en culture (alignements des vergers ou cercles de cultures), elles-mêmes circonscrites par les infrastructures d'irrigation (pivot, tuyaux de goutte-à-goutte, forage). Ils résultent d'initiatives pionnières individuelles ou sociétaires, rendues en partie possibles grâce à diverses formes de clientélisme politique, notamment pour l'accès au foncier et à l'eau. Depuis les années 2000, les marges désertiques de Wadi Natroun (ouest du Delta), de la dépression du Fayoum (moyenne Égypte) ainsi que les oasis du désert occidental se distinguent par ce type d'implantation foncière spontanée. Au Fayoum, c'est avant tout un processus de colonisation agricole par les paysans des anciennes terres voisines qui est à l'œuvre (Barnes, 2014). Wadi Natroun ou le pourtour de l'oasis de Siwa se caractérisent, quant à eux, par l'implantation de grands investisseurs, souvent urbains, qui font appel à des bureaux d'étude afin de s'assurer de la disponibilité et de la qualité de l'eau d'irrigation. Les grandes entreprises agricoles s'appuient généralement sur une équipe restreinte d'ingénieurs agronomes et de commerciaux pour le succès de leur implantation dans de nouveaux secteurs désertiques. La capacité d'accès à la terre et à l'eau, les capitaux requis pour financer les infrastructures d'irrigation et de production, le délai d'attente avant l'entrée en production des vergers, ou encore les contraintes logistiques liées à l'éloignement et à la chaleur sont autant d'éléments qui sélectionnent les acteurs productifs et déterminent le devenir de ces avant-postes. C'est le cas du secteur de Wadi Natroun (illustration 8), où coexistent les oliveraies de très grandes entreprises dominant le secteur et celles de petits investisseurs urbains à la recherche d'un revenu complémentaire et confrontés à des contraintes et des coûts d'entrée très élevés.

Ces avants-postes constituent par ailleurs des lieux majeurs de spéculation foncière, un certain nombre d'investisseurs faisant le pari de l'intégration de ces espaces aux dynamiques de mise en valeur (agricole et/ou résidentielle) et donc de la possibilité de revendre à bon prix à plus ou moins long-terme.

\section{Les agro-parks : des plates-formes productives a-rurales?}

La projection de nouveaux modèles productifs et socio-spatiaux au sein des terres désertiques est une constante depuis les premiers projets nassériens et la mise en place de vastes fermes étatiques. Elle se poursuit dans les stratégies les plus contemporaines tout en se renouvelant, avec notamment des projets de clusters ${ }^{7}$ agricoles (Calas, 2013). L'objectif est de combiner bonification des terres, exploitation des eaux souterraines à grande profondeur, innovations productives et nouveaux modèles résidentiels créés exnihilo, largement inspirés de modèles d'habitat urbain. En témoigne ce plan dédié à l'aménagement du secteur désertique de Al-Moghra (Nord-Ouest du désert occidental), associant fonctions agricole, piscicole, logistique, industrielle et résidentielle (illustration 9). 


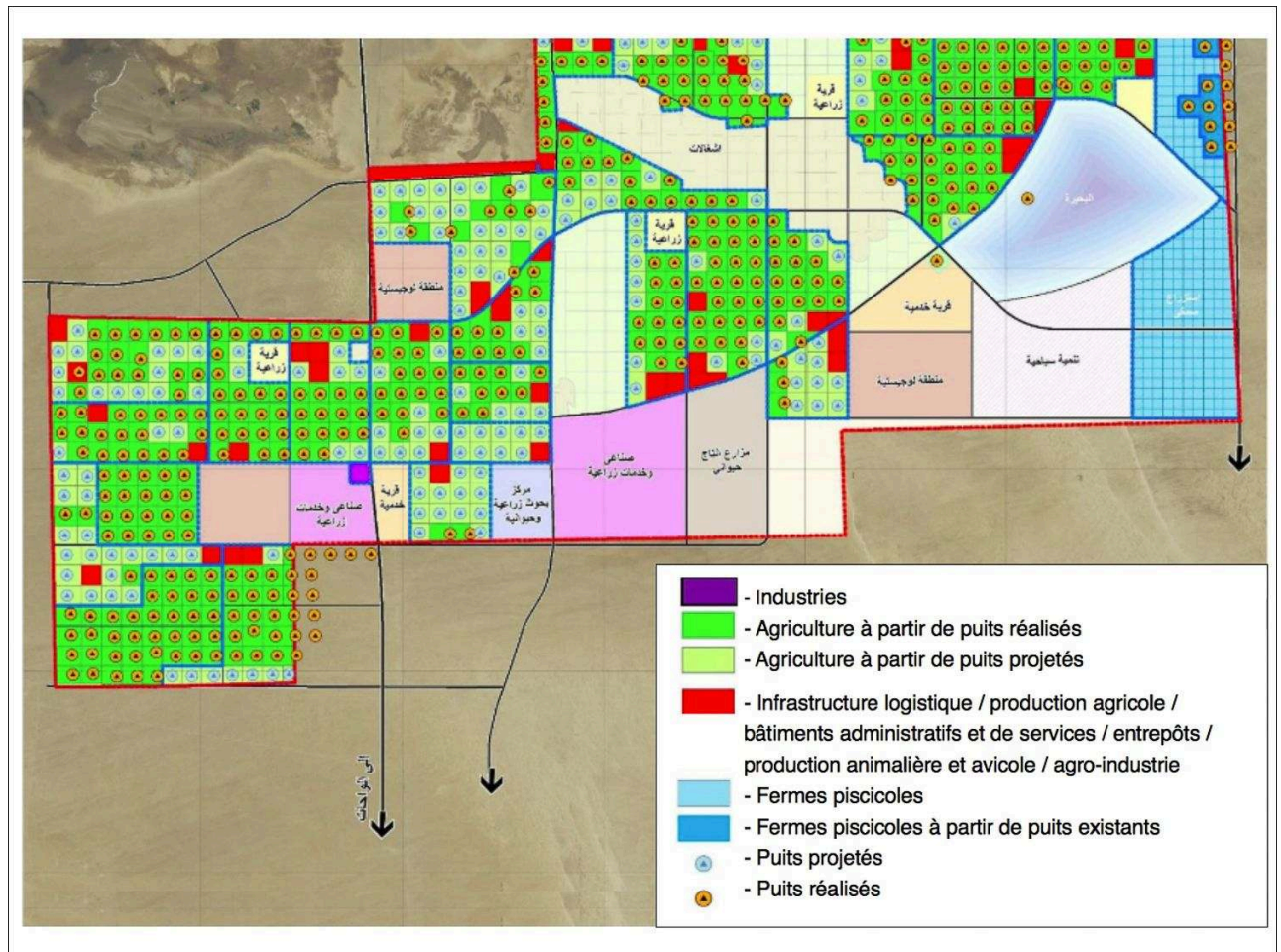

Source : http://www.elreefelmasry.com/, consulté le 26/01/2018.

C'est aussi le cas d'un projet de coopération entre l'Egypte et la Corée du Sud, via la Korea-Arab Society ${ }^{8}$, qui prévoit la construction d'une "ville agricole intégrée » sur une superficie de 300000 feddans dans la dépression désertique de Qattara au Nord-Ouest du pays. "Construit par des ouvriers égyptiens sous le contrôle d'experts coréens ", ce nouveau secteur est censé accueillir les toutes dernières technologies et innovations : serres intelligentes, usines de dessalement et centrales solaires, production de stevia (Arab Finance, 2017). On peut ici faire le lien avec les rural stations, décrites par R. Bourgeois (2015) dans son article consacré aux scénarios d'évolution des espaces ruraux de la planète. Il les définit comme des « complexes agro-industriels employant un nombre limité d'ouvriers, qui sont logés à proximité » (Bourgeois, 2015, p. 182). Ces complexes sont décrits comme "hautement spécialisés, spatialement isolés, mais hyper-connectés aux marchés via des axes routiers, ferroviaires et maritimes » (Bourgeois, 2015, p. 182). Les documents de planification et les projets de plates-formes agro-productives égyptiennes se caractérisent en effet par un rapport fonctionnel, productif et extractiviste à l'espace et à ses ressources foncières et hydriques. L'identité sociale et les rapports sociaux y sont envisagés avant tout par le rôle et la contribution au système agro-productif, plutôt que par des sociabilités, des modes d'habiter ou encore des pratiques quotidiennes. Rompant avec le terme rural employé par R. Bourgeois, ces modèles relèvent ainsi bien davantage d'agro-parks ou agro-clusters, associés à de grands projets logistiques, et se rapprochent d'un modèle urbano-agricole bien plus que rural. Ils s'inscrivent plus largement dans une forme d'agriculture «aterritorialisée " (Guibert, 2013), que nous pouvons définir de la façon suivante: l'activité de production agricole mise en œuvre sur un substrat vierge, sans relation ou presque avec la société, l'histoire et le patrimoine ruraux du pays ou de la région, ceci en raison de l'éloignement physique, de la discontinuité géographique, des 
technologies mises en œuvre réduisant au maximum la main-d'œuvre, ou encore de la nature non-traditionnelle des productions et de leur destination à l'export (ou vers l'industrie).

\section{Conclusion}

En lien avec la typologie des territoires agro-désertiques qui vient d'être proposée, on peut conclure en distinguant deux modèles spatiaux associés aux espaces du front pionnier égyptien : le modèle de l'entre-deux (Le Gall et Rougé, 2014 ; Acloque, 2014), d'une part, et celui de l'archipel ${ }^{9}$, d'autre part. Le modèle de l'entre-deux s'applique à l'ensemble géographique constitué des « anciennes » nouvelles terres, des «nouvelles » nouvelles terres et de différents avant-postes des marges arides du Delta. Il s'agit d'un espace d'interface entre vallée et désert, où des processus de construction et de maturation territoriales sont à l'œuvre. Cet entre-deux, qui regroupe la majorité des espaces bonifiés depuis les années 1950, a permis l'extension des terres du delta de $20 \%$ environ. Il s'est déplacé et continue à se déplacer à des rythmes inégaux en fonction des projets gouvernementaux et individuels et de leurs résultats respectifs. Les terres les plus anciennement bonifiées sont aujourd'hui intégrées - par leurs paysages, leurs fonctions et leurs modalités d'organisation - aux territoires historiques voisins, alors que des marges désertiques de plus en plus éloignées sont rejointes par cet entre-deux en translation.

Second modèle spatial: celui de l'archipel indissociable de la stratégie agricole contemporaine de bonification d'une pluralité d'îlots, en partie à l'état de projets et dispersés à l'échelle nationale. Ce modèle d'agriculture d'archipel est ainsi venu s'ajouter au précédent, sans toutefois s'y substituer puisqu'il coexiste, depuis les années 1990, avec l'entre-deux en expansion. Les projets d'agro-parks, pensés comme des vitrines de l'innovation et de la modernité agricole, en sont les plus emblématiques. Si ces territoires archipélagiques recouvrent en partie les espaces oasiens traditionnels, certains secteurs se caractérisent par une discontinuité forte vis-à-vis des espaces de peuplement, à l'instar du secteur de Sharq el-Oweinat à l'extrême sud du pays, véritable plate-forme agricole créée ex-nihilo dont l'accès est extrêmement discriminant du point de vue financier et logistique, mais aussi politique en raison du caractère stratégique des espaces désertiques frontaliers.

\section{BIBLIOGRAPHIE}

Aboulnaga A. et al., 2017. Study on small-scale family farming in the near East and North Africa region. Focus country Egypte. Le Caire, CIRAD-CIHEAM-FAO.

Acloque Desmulier D., 2014. Entre Delta du Nil et désert. Front pionnier agricole et recomposition territoriale en Egypte. Carnets de géographes [En ligne], ${ }^{\circ} 7$. URL: http://

journals.openedition.org/cdg/499 - DOI: https://doi.org/10.4000/cdg.499 
Akram-Lodhi A.H., Kay K. (ed.), 2009. Peasants and globalization: Political economy, rural transformation and the agrarian question. London, Routledge.

Alary V. et al., 2018. Desert land reclamation programs and family land dynamics in the Western Desert of the Nile Delta (Egypt), 1960-2010. World Development, vol. 104, p. 140-153.

Albaladejo C., 2012. Les transformations de l'espace rural pampéen face à la mondialisation. Annales de Géographie, n 686, p. 387-409.

Al-Beltagi A., Abu Hadid E., 2008. Al-raka'iz al-asasia lil-barnamig al-qawmi li-t'athim al-istifada min al-mai fi al-aradi al-qadima. Le Caire, Ministère de l'Agriculture.

Arab Finance, 2017. South Korea to build \$10bln integrated agricultural city in Egypt. Reproduit sur le site Farmlandgrab [En ligne]. URL: https://www.farmlandgrab.org/post/view/27399south-korea-to-build-10bln-integrated-agricultural-city-in-egypt (consulté le 24/08/2017)

Araghi F., 2009. The invisible hand and the visible foot: peasants, dispossession and globalization. In Akram-Lodhi A.H., Kay C. (ed.), Peasants and Globalization: Political economy, rural transformation and the agrarian question. Londres et New York, Routledge.

Arnauld de Sartre X., 2006. Fronts pionniers d'Amazonie, les dynamiques paysannes au Brésil. Paris, CNRS Editions, coll. Espaces \& Milieux

Arrault J.-B., 2005. Du toponyme au concept ? Usages et significations du terme archipel en géographie et dans les sciences sociales. L'Espace géographique, vol. 34, $\mathrm{n}^{\circ}$ 4, p. 315-328.

Ayeb H., 2010. La crise de la société rurale en Egypte ou la fin du fellah ? Paris, Karthala.

Ayrout H., 1938. Mœurs et coutumes des fellahs. Paris, Payot.

Banque mondiale, 2006. Egypt public land management, Policy Note (vol. 1 \& 2). Washington DC.

Barnes J., 2014. Cultivating the Nile. The everyday politics of water in Egypt. Duke Univ. Press.

Bernstein H., 2001. The peasantry in global capitalism: who, where and why? The Socialist Register, p. 25-51.

Bourgeois R., 2015. What Future for Rural Areas? Seven plausible rural transformations. Development, vol. 2-3, n 58, p. 177-186.

Calas B., 2013. Mondialisation, clusterisation et recyclage colonial. EchoGéo [En ligne], n² 26. URL: http://journals.openedition.org/echogeo/13586 - DOI: https://doi.org/10.4000/echogeo.13586

Critchfield R., 1978. Shahhat, an Egyptian. University of Syracuse Press

Demangeon A., 1932. Pionniers et fronts de colonisation. Annales de géographie, vol. 41, n 234, p. 631-636.

EEDC, 2015. Egyptian Economic Development Conference Brochure. Sharm el-Sheikh

Fanchette S., 1997. Le Delta du Nil. Densités de populations et urbanisation des campagnes. Tours, Fascicule de recherche URBAMA, $\mathrm{n}^{\circ} 32$.

Guibert M., 2013. Les formes associatives de production agricole en Argentine et en Uruguay : entre territoire et réseau ? Etudes rurales [En ligne], n 191. URL: http://journals.openedition.org/ etudesrurales/9786 - DOI: https://doi.org/10.4000/etudesrurales.9786

Guibert M., Bühler E.-A., Requier-Desjardins D., 2015. Entreprises agricoles et globalisation des espaces ruraux. Éclairages à partir de l'Argentine, du Brésil et de l'Uruguay. Géographie, économie, société, vol. 17, n 4, p. 387-408. 
Ireton F., 2011. Économie politique de l'agriculture : de l'encadrement étatique à la déréglementation. In Battesti V., Ireton F. (dir.), L'Égypte au présent. Paris, Sindbad, Actes Sud.

Kassim Y. et al., 2018. An agricultural policy review of Egypt: First steps towards a new strategy. MENA Working Paper IFPRI, $\mathrm{n}^{\circ} 11$, Washington DC-Le Caire.

Le Gall J., Rougé L., 2014. Oser les entre-deux ! Carnets de géographes [En ligne], n7. URL: http:// journals.openedition.org/cdg/496 - DOI: https://doi.org/10.4000/cdg.496

Lévy J., Lussault M. (dir.), 2003. Dictionnaire de la géographie et de l'espace des sociétés. Paris, Belin.

Margetic C., Rouget N., 2019. Ruralité. Hypergéo [encyclopédie ouverte]. https://

www.hypergeo.eu/spip.php?article740c (consulté le 16/09/2020)

Marshall A., 2009. S'approprier le désert. Agriculture mondialisée et dynamiques socio-environnementales sur le piémont côtier du Pérou. Le cas des oasis de Virú et d'Ica-Villacuri. Université Paris 1, Thèse de doctorat.

Martinez-Vazquez J., Timofeev A., 2011. Decentralizing Egypt: Not Just Another Economic Reform, in Decentralization in Developing Countries. Cheltenham, Edward Elgar.

Mathieu N., 2014. Mode d'habiter : un concept à l'essai pour penser les interactions hommesmilieux. In Chenorkian R. (ed.), Les interactions hommes-milieux. Éditions Quæ, p. 97-130.

Mayfield J. B., 2012. Field of Reeds, Social, Economic and Political Change in Rural Egypt: In Search of Civil Society and Good Governance. AuthorHouse.

Mitchell T., 2002. Rule of Experts: Egypt, Techno-Politics, Modernity. University of California Press.

Monbeig P., 1952. Pionniers et planteurs de São Paulo. Cahiers de la fondation nationale des sciences politiques, $\mathrm{n}^{\circ} 28$, Paris, A. Colin.

Morgan K., Marsden T., Murdoch J., 2008. Worlds of Food. Place, Power, and Provenance in the Food Chain. Oxford University Press.

Najjar D., 2013. The Money of Qaroon and the Patience of Ayoub: Women and Land in Egypt's Mubarak Resettlement Scheme (PhD). University of Western Ontario.

Pesche D., Losch B., Imbernon J. (dir.), 2016. Une nouvelle ruralité émergente - Regards croisés sur les transformations rurales africaines. Nepad-Cirad.

Rieutort L., 2009. Dynamiques rurales françaises et re-territorialisation de l'agriculture.

L'Information géographique, vol. 73, $\mathrm{n}^{\circ}$ 1, p. 30-48.

Rieutort L., 2012. Du rural aux nouvelles ruralités. Revue internationale d'éducation de Sèvres, vol. 59, p. 43-52.

Saad R., 2000. Agriculture and politics in contemporary Egypt: the 1997 tenancy crisis. Cairo Papers in Social Science, vol. 22, $\mathrm{n}^{\circ}$ 4, p. 22-35.

Scott J., 1998. Seeing like a state. How certain schemes to improve the human condition have failed. Yale University Press.

Sims D., 2014. Egypt's desert dreams. Cairo, AUC Press

Sowers J., 2011. Remapping the Nation. Critiquing the state, Environmental narratives and desert land reclamation, in Egypt. In Davis D., Burke T. (ed.), Environmental Imaginaries of the Middle East. Athens, Ohio University Press.

Svampa M., 2019. Neo-extractivism turn in Latin America. Cambridge University Press 
Théry H.,1976. Rondônia, mutations d'un territoire fédéral en Amazonie brésilienne. Paris 1, Thèse de 3ème cycle.

Van der Ploeg J.D., 2018. From de- to repeasantization: The modernization of agriculture revisited. Journal of Rural Studies, vol. 61, p. 236-243.

\section{NOTES}

1. Selon R. Saad (2000, p. 24), sur un million de personnes, incluant les locataires évincés et l'ensemble de leurs familles, seuls 1,5\% d'entre-eux auraient en réalité obtenu des terres dans un secteur de bonification.

2. Un feddan est une unité de surface correspondant à 0,42 ha.

3. Nous ne pouvons pas développer, dans le cadre de cet article, la question des modalités d'accès au foncier désertique caractérisées par un manque de transparence et une forte dimension clientéliste.

4. Le CAPMAS (Central Agency for Public Mobilization and Statistics) est l'institution nationale des statistiques en Egypte.

5. Les exportations de produits agricoles ont été multipliées par quatre entre 2001 et 2015, passant de 500 millions à 2,1 milliards de \$ (Agence Ecofin, 3/02/2016).

6. Dans le cas du markaz de West Nubaria (Behera), qui se situe au cœur des périmètres bonifiés les plus récents, l'évolution démographique est très significative, passant de 885 habitants à plus de 100000 sur la même période (URL: https://www.citypopulation.de/php/egypt-admin.php? adm1id=18, consulté le 21/08/2019)

7. Le terme cluster est un terme flou et large utilisé ici pour désigner tout regroupement d'acteurs du secteur agricole, souvent localisés dans un même secteur géographique et nouant entre-eux des relations étroites de production et de commercialisation.

8. The Korea-Arab Society (KAS) is a non-profit and an incorporated foundation, funded by Korean and Arab governments. The bilateral cooperation between Korean and 22 Arab countries will enhance mutual development in diverse areas not only in economy but also in arts, culture, sports and academia. URL: http://eng.korea-arab.org/?c=user\&mcd=foundation, consulté le 22/01/2018

9. Le terme "archipel » est devenu une notion et même un concept de la géographie et des sciences sociales, qui a été décliné dans une grande diversité de domaines (Arrault, 2005).

\section{RÉSUMÉS}

Depuis le milieu du XX $\mathrm{X}^{\mathrm{e}}$ siècle, les périphéries désertiques égyptiennes sont devenues le lieu de projection de l'avenir de l'agriculture nationale. Une véritable frontière agro-désertique a émergé, se déplaçant à des rythmes inégaux au cours des six dernières décennies. Cet article se propose d'éclairer la nature des nouveaux territoires agro-désertiques égyptiens et de comprendre en quoi ils interrogent les catégories de rural et de ruralité. La question du rapport au territoire de la nouvelle agriculture et de ses acteurs est posée, ceci depuis les parcelles de petits paysans au sein de villages créés ex-nihilo jusqu'aux exploitations agro-capitalistes contemporaines, parfois distantes de plusieurs centaines de kilomètres des principaux espaces de peuplement. Faut-il s'affranchir du concept de ruralité pour rendre compte des espaces du front 
pionnier égyptien? Comment penser l'agricole sans rural et quelles catégories construire pour caractériser ces néo-territoires agro-désertiques?

Since the middle of the 20th century, desert margins have become the place where the future of the Egyptian agriculture is projected, both by the state and by international institutions. This article sheds light on the nature of the new agro-desert territories and shows how the frontier questions the very categories of rural and rurality. We discuss how this expanding agricultural sector and its diversity of stakeholders (from small peasants to large companies) are spatially embedded in desert areas. To what extent the analysis of the Egyptian agricultural frontier should go beyond the concept of rurality? What socio-spatial categories should be used and built to characterize the new territories?

\section{INDEX}

Mots-clés : front pionnier, agriculture, espace aride, rural, ruralité, Egypte

Keywords : frontier, agriculture, arid area, rural community, rurality, Egypt

Thèmes : Sur le Champ - Sur le Terrain

\section{AUTEUR}

\section{DELPHINE ACLOQUE}

Delphine Acloque, delphine.acloque@gmail.com, est chercheure postdoctorante associée au CEDEJ (Le Caire) et au laboratoire CITERES (Tours). 